\title{
Egg Shell and Nano Technology a Veritable Combination to Promote Regeneration of Bone in Intrabony Defect: A Case Report
}

\author{
Dr. Asha $\mathrm{A}^{\mathbf{1}^{*}}$, Dr. Rashmi $\mathbf{P}^{2}$, Dr. MLV Prabhuji ${ }^{3}$, Dr. Rathana $\mathbf{M}^{\mathbf{1}}$ \\ ${ }^{I}$ PG in Dept. of Periodontics, Krishnadevaraya College of Dental Sciences and Hospital, Sir MVIT Campus, \\ New airport road, Bengaluru- 562157, India \\ ${ }^{2}$ Reader, Dept. of Periodontics, Krishnadevaraya College of Dental Sciences and Hospital, Sir MVIT Campus, \\ New airport road Bengaluru- 562157, India \\ ${ }^{3}$ Professor and HOD, Dept. of Periodontics, Krishnadevaraya College of Dental Sciences and Hospital, Sir \\ MVIT Campus, New airport road Bengaluru- 562157, India
}

*Corresponding Author: Dr. Asha A, Dept. of Periodontics, Krishnadevaraya College of Dental Sciences, Hunsemaranhalli, New Airport Road, Bengaluru - 562157, India

\begin{abstract}
Introduction: The search for an ideal graft material to reach the goal of complete bone regeneration is never ending. Each of the graft material whether autograft or allograft have some of the ideal characteristics like osteoconductivity, osteinductivity and osteopromotivity. Hydroxyapatite is a naturally occurring mineral component of bone has osteoconductive properties and is a stable fraction of most alloplast. The egg shell of the hen was used as a source to procure hydroxyapatite with nano processing.
\end{abstract}

Objective: The objective of this study was to evaluate efficacy of eggshell derived hydroxyapatite (EHA) for regeneration of bone in intrabony defects.

Materials and Methods: A 5lyrs old male patient without any systemic complications or allergic reaction reporting to outpatient department of Krishnadevaraya College of dental science was thoroughly examined for any intraoral periodontal findings. After ascertaining the presence of intrabony defect with cone beam computer tomography (CBCT) he was enlisted for surgery (open flap debridement). The hen's egg shell was subjected to various steps using nano technology in biochemistry dept. KCDS\&H. All the sterilization protocol was followed and the sample was subjected to in vitro cytotoxicity and scanning electron microscopy (SEM) analysis was carried out. Post-operative surgical protocol (1week, 1 month) was followed. Postoperative clinical and radiographic (RVG with grid) assessments were carried out at 1 month, 3 months and 6 months.

Results: Post-operative complications were not seen and healing was uneventful. By the end of the six months, the defects grafted with EHA showed a very good bone formation.

Conclusion: Egg shell derived nano hydroxyapatite bone graft could be an important tool for periodontal bone regeneration.

Abbreviation: EnHA - Egg shell derived nano hydroxyapatite

Keywords: Egg shells, Alloplast, Intra-bony defects, Osteoconductivity, Cytotoxicity

\section{INTRODUCTION}

The most elusive goal of periodontal therapy is the regeneration of periodontium after episodes of destructive periodontal diseases. To reach this goal a wide variety of bone replacement grafts have been tried with a varying degree of success. The characteristics of a model bone graft would be faclilitation of osteogenesis, low risk of infection, ready availability, low antigenicity and a high degree of success.
Among regenerative grafts, autografts is considered as a gold standard. However autografts have their own set of disadvantages, namely use of a second surgical site, prolonged treatment time, increased surgical complications, Pain dysfunction of the harvested and high operative cost.

The other options would be allografts and xenografts, which again lead to immunologic reactions, infections and improper fibrous 
healing. Thus the use of alloplast or synthetic bone graft substitutes has gained popularity, over other sub-groups of biomaterials.

Hydroxyapatite (HA) is an inorganic component of hard tissues and is one of the most ubiquitous biomaterials for reconstruction of bone so far. It is osteo-conductive with few studies even claiming an osteo-inductive property when used in a nano-crystalline form. This versatile material is derived from many sources such as bovine bone, fish bone or coral.

The most prominent quality of HA is its high level of bioactivity and ability to quickly integrate with bone. This bioactivity could be attributed to its ability to concentrate active fibrin on its surface according to our recent study. Any regeneration of periodontium would require the presence of fibrin-fibronectin system to allow cementum formation. The HA available for clinical usage is either in porous or dense block/granular form. By nature it is an inorganic ceramic substance and thus there is no chance for foreign body reaction. Its porous nature allows the in growth of bone into it and thereby merging with the native bone.

The commercially available HA has a high costing due to the usage of purity reagents. Most of the HA derived from natural sources such as bovine bone, fish bone or coral has a varied physical and chemical properties of the species from which they are derived.

More recently, avian eggshell powder has been tentatively tried as a bone substitute. Its key components are calcium carbonate (94\%), calcium phosphate $(1 \%)$, organic matter $(4 \%)$ and magnesium carbonate (1\%). The egg shell as we know is easily available and is biodegradable as well. The mineral component of the avian egg shell provides reasonable thickness for preparing particulate matter of different sizes. The available literature points to its superior properties in comparison to the commercially available grafts, histomorphically as well as radiographically.

Thus the aim of the present case study was to assess all the aspects of bone regeneration in intrabony defects when eggshell powder was used as a bone substitute.

\section{CASE Report}

A 51years old male patient reported to the Department of periodontology Krishnadevaraya College of Dental Science and Hospital for a routine dental examination.
Upon radiographic examination the patient presented with a generalized crestal bone loss and a vertical defect irt 46. (Figure 1)

Clinically there was an intrabony pocket on the distal aspect of 46 with a Probing pocket depth of $10 \mathrm{~mm}$ measured using UNC 15 periodontal probe (University of North Carolina $\mathrm{Hu}-$ Friedy, Chicago, IL, USA)

There was also cervical abrasion irt 14, 15, 16.

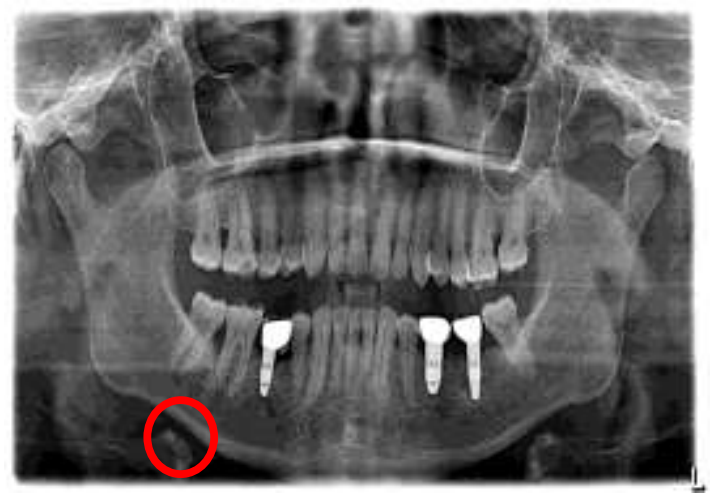

Figure1. (a) Per operative orthopantamograph showing intrabony effects irt 46 and 47

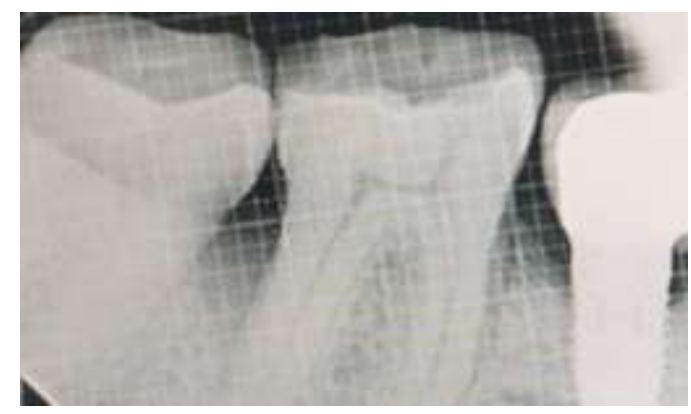

Figure1. (b) Preoperative RVG with Grid

\section{Materials And Methods}

\subsection{Egg-Shell Graft Preparation}

Eggshells were collected and immersed in boiling water for about 1 hour to remove the inner membrane and surface contaminants, later eggshell were strained and kept for drying for about 24 hours.

After drying, they were ground into a powder and immersed in sodium hypochlorite $(\mathrm{NaOCl})$ to remove organic components.

Then, they were extensively washed with Millipore water and dried in a vacuum oven for $5 \mathrm{hrs}$. at $110{ }^{\circ} \mathrm{C}$. So formed eggshell powder was converted into calcium- EDTA complex by reacting it with EDTA. This Ca- EDTA reacted with disodium hydrogen orthophosphate while maintaining the $\mathrm{pH}$ above 10 and is subjected to microwave irradiation to produce HA (Figure 2). 


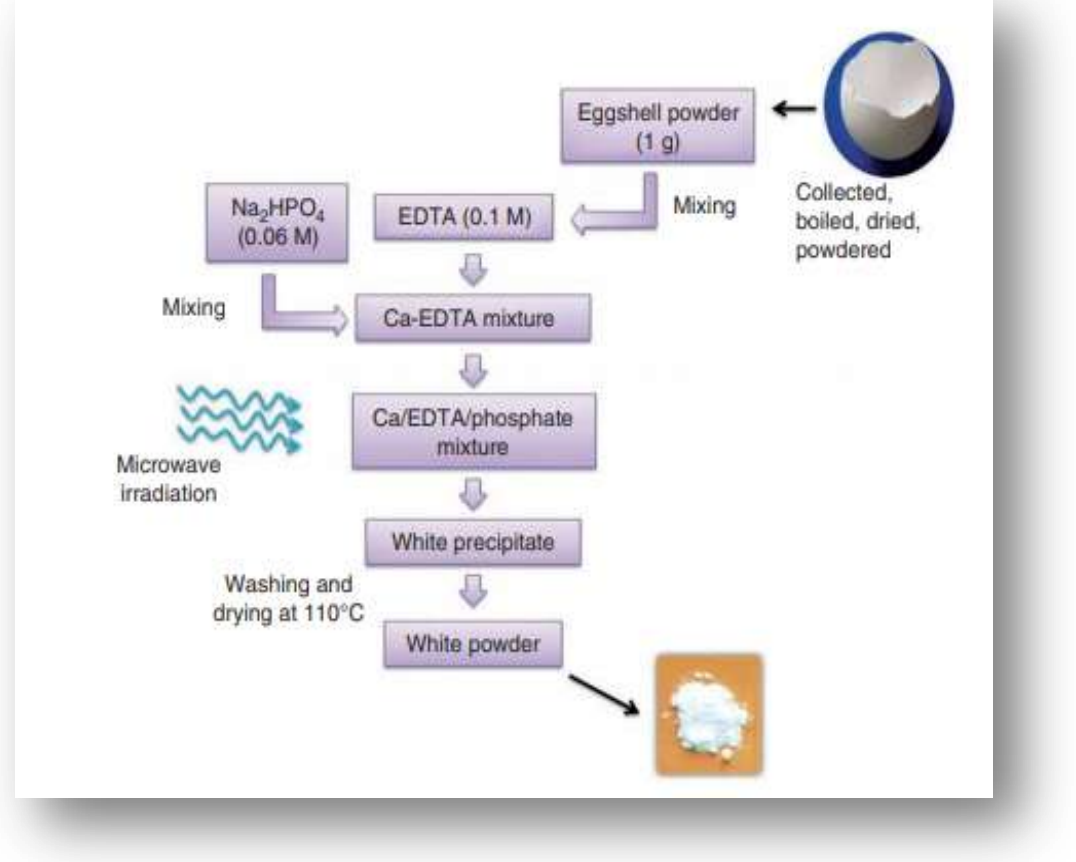

Figure2. Flowchart for the microwave conversion of eggshell powder into nanoHA

\subsection{Scanning Electron Microscopy (SEM)}

EnHA graft was assessed with scanning electronic microscopy (Indian Institute of Science [IISE] Bangalore) for uniformity and standardization of the particle size. Most of the graft particle size range from 1.012 to $2.37 \mu \mathrm{m}$ in length and 300 to $750 \mathrm{~nm}$ in width. (Figure 3)

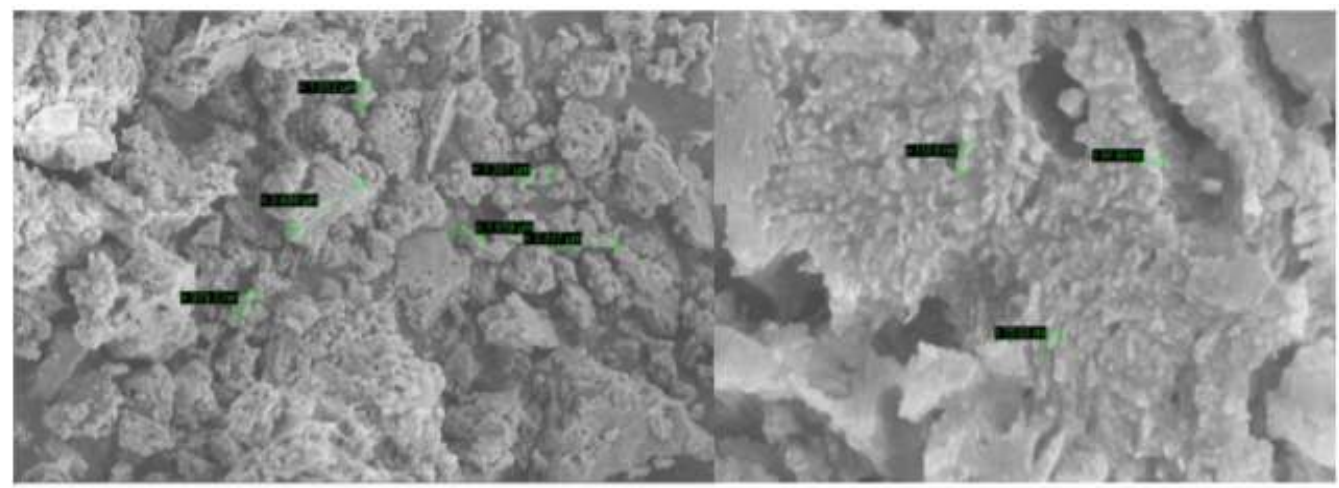

Figure.3 SEM analysis of EnHa graft particle size

\subsection{Pre-Procedural Evaluvations}

Routine periodontal parameters namely, Probing depth (PD), Clinical attachment level (CAL), Bleeding on probing (BOP), were recorded just before the surgery.

To ascertain the general health of the patient, basic blood investigation was carried out. Oral hygiene was evaluated and found to be satisfactory.

To increase the accuracy prior to treatment, the patient was subjected to sectional CBCT in the fourth quadrant and Radiovisiography with Grid was taken.

\subsection{Surgical Procedure}

A pre-procedural mouth rinse of chlorhexidine Gluconate $0.2 \%$ was given to the patient. Lignocaine hydrochloride (1:80000) was used as local anaesthetic and an inferior alveolar nerve block was given to anesthetise the operative area. A Sulcular / crevicular incision was given to preserve the maximum amount of keratinized tissue for regenerative procedure.

Flap reflection using Molts No.9 periosteal elevator was carried out. Upon reflection the presence of intrabony defect irt distobuccal aspect of 46 was elicited. A thorough 
Egg Shell and Nano Technology a Veritable Combination to Promote Regeneration of Bone in Intrabony Defect: A Case Report

debridement of the root surfaces and the defect was completed using scalers and curettes. (HuFriedy).The defect was filled with eggshell graft and condensed properly. A collagen membrane (HEALIGUIDE ${ }^{\circledR}$ - Advanced Biotech) was used to stabilize the graft. Flap was approximated using silk (3-0) suture with a simple interrupted pattern. (Figure 4, 5, 6, 7, 8, 9)

RVG was taken to ascertain the proper graft placement intra-operatively. (Figure 10)

Non-eugenol pack was placed and standard post-operative instructions were given Analgesics were prescribed for five days and chlorhexidine mouthwash was prescribed for fifteen days.

Patient was recalled after one week for suture removal. Follow-up was done for 1, 3, and 6 months. At 6 months the periodontal parameters namely PPD, CAL were recorded. A follow-up RVG with grid was done to evaluate bone gain.

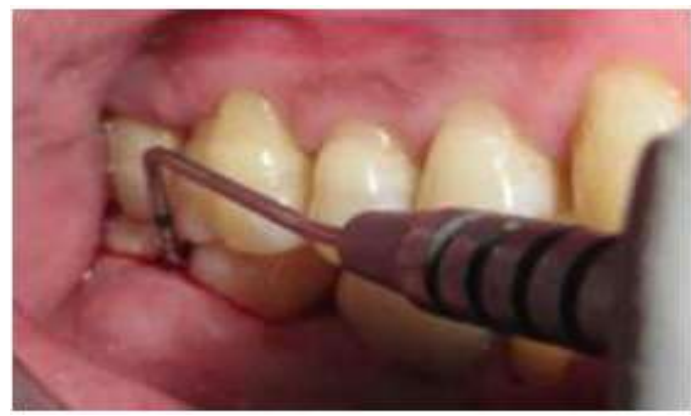

Figure4. Initial probing depth

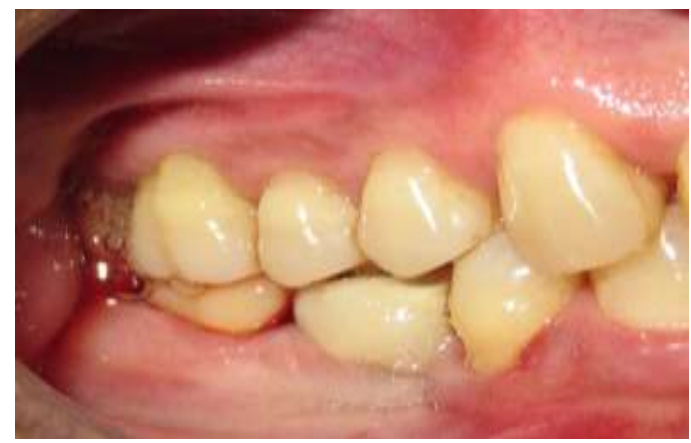

Figure5. Sulcular incision given

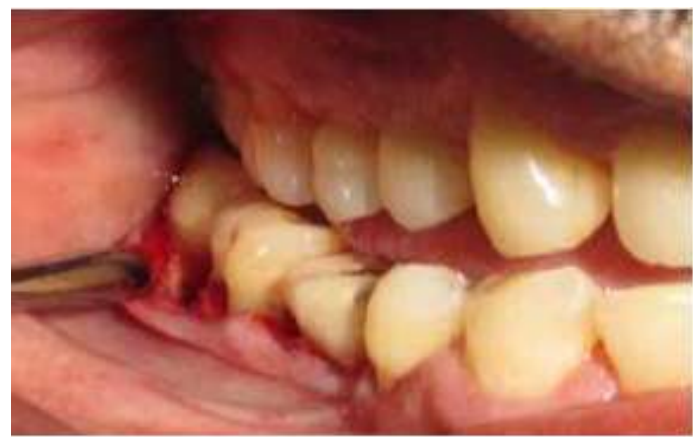

Figure6. Flap reflection

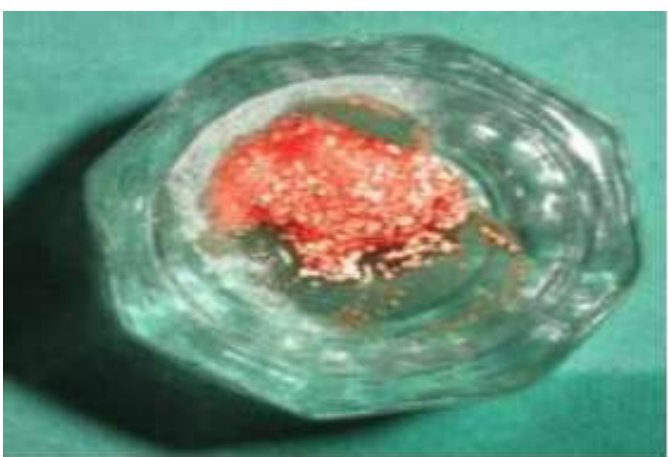

Figure7. Egg shell graft

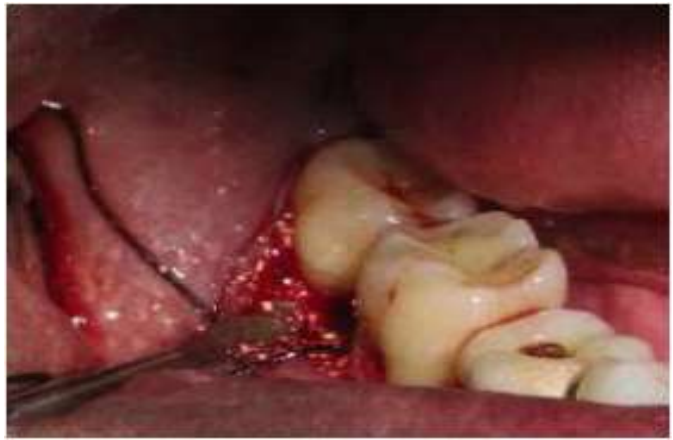

Figure8. Placement of EnHA graft material in defect site

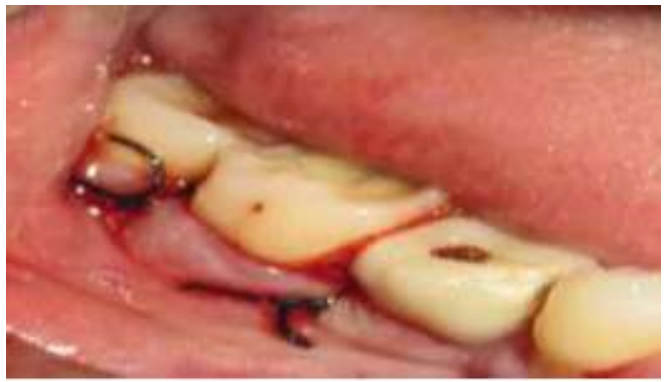

Figure9. Placement of simple interrupted suture

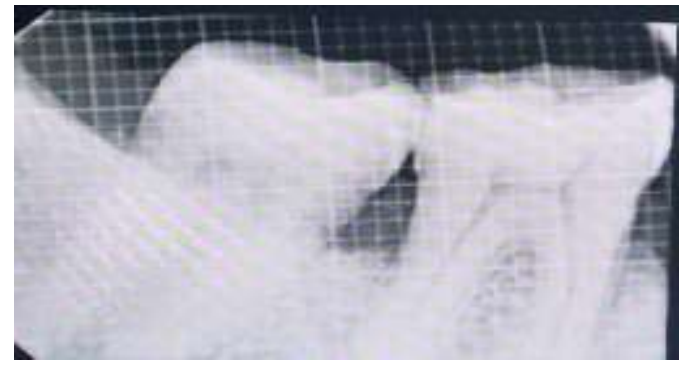

Figure10. $R V G$ taken after graft placement

\section{ReSUlts}

By the end of the six month, the defect grafted with EHA showed a complete bone formation. However, bone formation in non-grafted sites was insignificant. The level of density measurements were equal that of surrounding normal bone. These results indicate that the osseous regeneration of the bone defect filled with EHA is significant. (Figure 11, 12) 


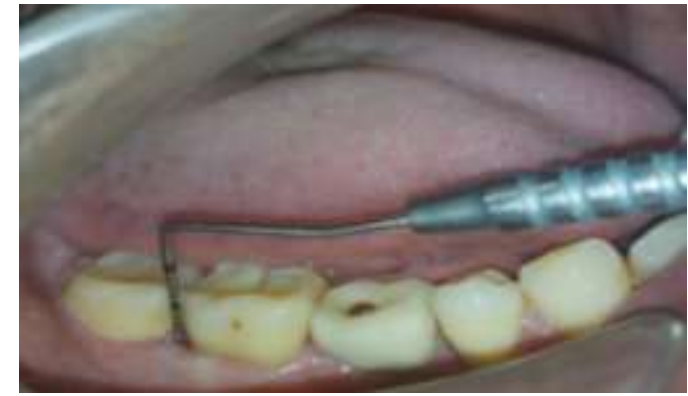

Figure11. 6 months post operative reduction in probing depth

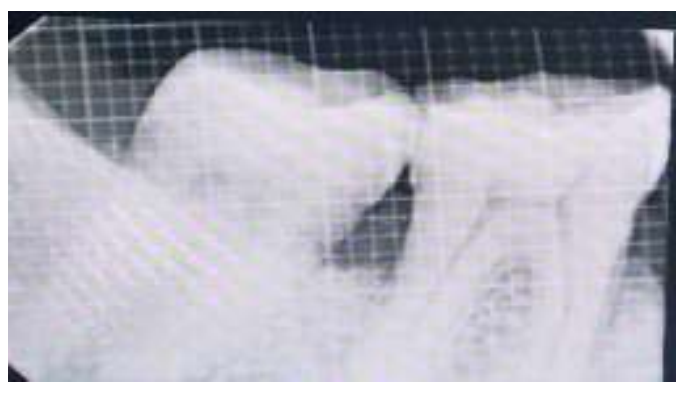

Figure12. 6 months post-operative gain in bone height

\section{DISCUSSION}

The never ending quest for the "Holy Grail "of periodontal regeneration namely "The ModelGraft" having osteoconductive, osteoinductive and osteogenicis still on. The advancement in material science and technology has definitely simplified the aspect of preparation of bone grafts. But the ideal biomaterial having optimal reconstructive ability and minimal bio hazardous property is still not anywhere on the horizon.

The commonest sequlae after periodontal disease is the loss of alveolar bone. The ideal defect to regenerate would be an intrabony defect. The regeneration of bone is dependent on elimination of periodontal infection thoroughly is paramount to the success of regenerative flap surgery.

The egg shell derived bone graft substitute is the simplest material to procure and the detailed preparation protocol helps to modify it into a fine nano crystalline form. Any probable adverse bio events are averted by the thorough cytotoxicity assay.

The preliminary report on use of egg shell bone substitute was carried out by Baliga et al. [1] in the cystic cavity of the jaw and showed moderate degree of bone regeneration though the osteo-inductivity could not be confirmed.
Another study by Dupoiorirex L et al. [2] in rats concluded the feasibility of using egg-shell powder for bone fill and did not confirm osteoinductivity.

Improvisation in the synthesis of flower like nanocrystalline structure using rapid microwave irradiation with the help of EDTA was documented by Suresh kumar et al. [3, 4] and a similar protocol was used in our study in the preparation of egg-shell derived HA. He concluded that the high level of bioactivity of the prepared nano-hydroxyapatite may be due to the beneficial effects of magnesium as well as carbonates and nano-sized HA have better bioactivity than coarser crystals. It can also act as an effective carrier for drug delivery applications.

A series of studies by kattimani et al. [5] for the use of egg-shell derived nano hydroxyapatite as graft material after surgical removal of mandibular third molar showed that EnHA enhances the bone regeneration in defect site and the grafted sites maintained bone height.

In the current case report there was excellent improvement in clinical parameter i.e. probing pocket depth which reduced from $10 \mathrm{~mm}$ at base line to $5 \mathrm{~mm}$ at 6 months. Radio graphically there was a significant bone fill in the range of 3 to $4 \mathrm{~mm}$ at 6 months. For an accurate radiographic assessment $\mathrm{CBCT}$ of the defect site could not be procured due to the restricted access for medical care in the COVID-19 pandemic times. It can be said with a reasonable amount of conviction that the Egg shell derived nano hydroxyapatite has a greater scope for alveolar bone regeneration than it's contemporary biomaterials.

\section{CONClusion}

Regaining the lost bony architecture has always been an insurmountable goal. Most of the bone graft materials utilized so far have their own sets of pros and cons. The hen's egg-shell derived nano hydroxyapatite is the most easily available product and that too in any quantity that is required. These aspects will improve the feasibility of the material. The improvement in the key factors for periodontal regeneration in this case report denote that EnHA for treatment of intrabony defects is a viable option without an iota of doubt. 


\section{FUTURE DIRECTIONS}

Since it's a case report it should be followed by a multicentric study with a larger and diverse population in various periodontal defects to evaluate the efficacy of EnHA. A histomorphometric analysis may provide deeper understanding into the intricate components of bone regeneration and provide a more profound insight into the biomechanics of this indigenous graft.

\section{DECLARATION OF CONFLICTING INTERESTS}

The author(s) declared no potential conflicts of interest with respect to the research, authorship, and/or publication of this article.

\section{FUNDING}

None

\section{REFERENCES}

[1] Baliga M., Davies P., Dupoirieux L. Powdered eggshell in the repair of cystic cavities of the jaw. Preliminary study, Rev Stomatol Chir Maxillofac. 99(1), 86 (1998)
[2] Laurent Dupoirieux., Didier Pourquier., Frangois Souyris. Powdered eggshell: a pilot study on a new bone substitute for use in maxillofacial surgery, Journal of Cranio Maxillo-Facial Surgery. 23, 187 (1995)

[3] Kumar G Suresh., E K. Girija., "Flower-like hydroxyapatite nanostructure obtained from eggshell: A candidate for biomedical applications, Ceramics International. 39(7), 8293 (2013)

[4] G. Suresh Kumar., A. Thamizhavel., E.K. Girija., Microwave conversion of eggshells into flower-like hydroxyapatite nanostructure for biomedical applications, Materials Letters. 76, 198 (2012).

[5] Kattimani, Vivekanand., Krishna Prasad Lingamaneni., Samatha Yalamanchili., Muralidhar Mupparapu. "Use of eggshellderived nano-hydroxyapatite as novel bone graft substitute-A randomized controlled clinical study," Journal of biomaterials applications. 34(4), 597 (2019)

Citation: Dr. Asha A, Dr. Rashmi P, Dr. MLV Prabhuji, Dr. Rathana M. Egg Shell and Nano Technology a Veritable Combination to Promote Regeneration of Bone in Intrabony Defect: A Case Report. ARC Journal of Dental Science. 2020; 5(4):33-38. DOI: https://doi.org/10.20431/2456-0030.0504005.

Copyright: (C) 2020 Authors. This is an open-access article distributed under the terms of the Creative Commons Attribution License, which permits unrestricted use, distribution, and reproduction in any medium, provided the original author and source are credited. 